Zaza Katsarava

Maka Kukava

Ekaterine Mirvelashvili

Alexander Tavadze

Anna Dzagnidze

Mamuka Djibuti

Timothy J. Steiner

\section{A pilot methodological validation study for a population-based survey of the prevalences of migraine, tension-type headache and chronic daily headache in the country of Georgia}

Received: 23 January 2007

Accepted in revised form: 16 February 2007

Published online: 11 May 2007

Z. Katsarava ( $($ )

Department of Neurology,

University of Essen,

Hufelandstrasse 55, DE-45122 Essen,

Germany

e-mail: zaza.katsarava@uni-due.de

Tel.: +49-201-723-2467

Fax: +49-201-723-5919

M. Kukava • A. Dzagnidze Department of Neurology,

Tbilisi Medical University,

Tbilisi, Georgia

E. Mirvelashvili • M. Djibuti Department of Public Health, Tbilisi Medical University,

Tbilisi, Georgia

M. Djibuti • A. Tavadze

Partnership in Research and

Action for Health,

Tbilisi, Georgia

T.J. Steiner

Division of Neuroscience and Mental

Health, Imperial College

London, UK

\begin{abstract}
We report the methodology of an epidemiological survey of the prevalences of migraine, tension-type headache and chronic daily headache in Georgia.

Medical residents visited adjacent households in Tbilisi to interview a pre-defined target of 100 biologically unrelated subjects. All respondents reporting headache in the previous year, as well as random 20 non-headache controls, were examined by a neurologist. The response rate was $70 \%$. Of 156 respondents, 93 were biologically unrelated and 45 (48\%) reported headache in the previous year. Eight subjects fulfilled all IHS criteria for migraine (1-year prevalence $8.6 \%$ [95\% CI: $2.9-14.3 \%]$ ), and 13 had probable migraine, meeting all but the criterion for duration. Nineteen had tension-type headache $(20.4 \%$ [95\% CI: 12.2-28.6\%]) and five had chronic daily headache $(5.4 \%$ [95\% CI: 1-10.0\%]). In compar-
\end{abstract}

isons of diagnoses by questionnaire and neurologist (considered the gold standard), sensitivities for the questionnaire of $89 \%$ for migraine and $67 \%$ for tension-type headache were calculated (overall kappa=0.74).

\section{Introduction}

Primary headache disorders, principally migraine and tension-type headache, affect up to $80 \%$ of the general population and are among the most important public health problems throughout the world. Whilst the prevalence and socioeconomic impact of migraine, and to a lesser extent of tension-type headache, have been studied many times in the last 20 years, the majority of surveys have been performed in the developed countries of Western Europe and 
North America [1]. Only a few have been carried out in Eastern Europe, and no data are available from the countries of the former Soviet Union.

In recognition of the scale of the global problem, three major international headache non-governmental organisations, in collaboration with the World Health Organization (WHO), have committed to the initiative "Lifting The Burden: The Global Campaign to Reduce the Burden of Headache Worldwide" [2]. The Global Campaign aims, in its first stage, to fill the gaps in the knowledge of headache-related burden worldwide.

The International Headache Society (IHS) has established its Russian Linguistic Subcommittee, to promote knowledge of headache and encourage the interest of physicians in countries of the former Soviet Union. In a collaborative enterprise between these two, a working group has been formed of representatives from Armenia, Azerbaijan, Byelorussia, Georgia, Moldova and Russia which, as a primary goal, seeks to perform a burden-of-illness study of headache in these countries. A successful epidemiological study will provide data on prevalence and socioeconomic impact of the important headache disorders in the general populations of these countries as a prelude to planning and implementing, with local health policy makers and the Global Campaign, health-care programmes for headache where none currently exist.

The infrastructure of Georgia is such that neither telephone nor postal surveys are likely to be effective, and it is necessary to carry out such a study by interviews conducted through door-to-door visits. The method requires testing in a pilot study before use in a full survey, and this is reported here. The aim was to validate methodology that could be used for epidemiological studies of headache in all countries of the former Soviet Union and possibly other developing countries worldwide.

\section{Patients and methods}

The study protocol was approved by the Georgian National Council on Bioethics.

\section{Questionnaire}

We adapted a questionnaire that had been used and validated for epidemiological headache research in Germany [3]. It was translated from German into the Georgian language by ZK. It had four parts: (a) personal data; (b) a medical enquiry, including questions related to migraine, tension-type headache, trigeminal autonomic cephalalgias and the use of acute and preventative headache medication as well as any other medications; (c) sociodemographic status evaluation; and (d) enquiry into willingness to pay (WTP) for acute and preventative headache medication. The medical enquiry began with a screening question: "Have you had headache last year not related to flu, hangover, cold or head injury?", as recommended by earlier studies $[4,5]$. Subjects who responded "yes" to this question were asked to complete the questionnaire. Subjects who responded "no" were asked to respond to the demographic and general medical questions only. Questions relating to migraine, tension-type headache and trigeminal autonomic cephalalgias were based on IHS diagnostic criteria [6]. From the German experience we expected to achieve high sensitivity and specificity for migraine and tension-type headache but be able only to screen for possible cluster headache [7]. The questions on WTP were asked according to the bidding game method [8]. Briefly, they asked subjects with headache how much money they would spend per month for an effective medication package defined as medication achieving "very good" pain relief for acute headache coupled with preventative medication reducing headache frequency by more than one half. The bidding began by first asking whether the individual would pay 15 Georgian Lari (GEL, approximately USD 7.5) for the package. If the answer was "yes", the interviewer incremented the bid in steps of GEL 5 until the answer was "no". The last sum of money receiving a "yes" response was then the WTP result from this bidding game approach. If the initial answer was "no", the interviewer reduced the bid by GEL 5 and continued this process until the respondent said "yes". The first sum of money receiving a "yes" response was the WTP result in these cases.

As a first step, the questionnaire was presented to a few available patients with migraine and tension-type headache seen in the Department of Neurology of the Tbilisi Medical Academy. As no headache outpatient clinic existed in Georgia, we had no possibility of testing the questionnaire more thoroughly in an outpatient setting. We therefore decided to perform the validation during the pilot epidemiological study.

Pilot epidemiological study

The total population of the Republic of Georgia in 2000 was 4,435,200 inhabitants, 53\% urban and 47\% rural, with 1080000 inhabitants in the capital city, Tbilisi. The study was conducted in two districts of Tbilisi that were representative for demographic structure of the entire population of the city and occupied by multi-storey apartment buildings with 40-100 households per building.

We used the "cold-calling" method of door-to-door survey (calling unannounced), visiting adjacent front doors of residential buildings. This decision was based on the following considerations: (a) a door-to-door survey performed by medical residents would ensure a high quality of collected information; (b) the low income levels in Georgia made it feasible to provide salaries for medical residents engaged for the task; (c) participation in a study organised by IHS would be encouraging for young physicians and ensure high levels of enthusiasm and therefore high methodological quality; (d) lack of infrastructure (e.g., functioning postal service, telephone lists, etc.) made other methods impossible.

Three medical residents performed the door-to-door survey. 
Consecutive adjacent households were contacted until a predefined target number of 100 biologically non-related subjects was achieved. In cases of refusal, subjects were considered as non-responders. If nobody opened the door, surveyors returned once. If nobody responded for the second time, the adjacent household was contacted in its place.

We differentiated between the main and secondary members of the household: husband and wife, together with any other biologically unrelated adults, were considered as the main study population, while children, grandmothers, grandfathers, aunts, uncles and other relatives were secondary household members. All main and secondary adult family members were interviewed by surveyors using the standard headache questionnaire. Data from the main study population were included in the primary analysis to calculate the prevalence and burden of headache in the general population. Data from both main and secondary adult study populations were used for validation of the questionnaire.

Each subject who reported headache not related to flu, hangover, cold or head injury in the previous year was invited for neurological interview and examination which were performed by one of two neurologists who had trained in the field of headache for two years at the Department for Neurology, University of Essen. At this interview, primary headache disorders were diagnosed according to IHS criteria and, when necessary, symptomatic headaches were excluded by neurological examination and cranial CT/MRI. Of respondents not reporting headache, 20 were randomly selected and also invited for neurological examination. The neurologists were not aware of any responses to the questionnaire.

\section{Analysis}

Data analysis was performed by SPSS 13.0 and BiAS 8.0. Comparisons of interval-scaled variables were made using Student's t-test and of ordinal-scaled variables using the chisquared test. The level of significance was set at $p<0.05$.

Sensitivity and specificity of the questionnaires for migraine and tension-type and for the combination of migraine and tension-type headache were calculated by comparing questionnairebased diagnoses with the diagnoses made at neurological interview (physician diagnoses), the latter being considered the gold standard. Cohen's kappa was calculated for the overall agreement of questionnaire- and physician-diagnoses.

Crude prevalences of migraine, tension-type headache and chronic daily headache, with $95 \%$ confidence intervals (CI), were calculated as recommended by Bortz [9].

\section{Results}

Seventy adjacent households were contacted. Of these, 49 households $(70 \%)$ were interviewed whilst 21 refused. Individuals who refused the interview tended to be younger $(42.1 \pm 10.0$ vs. $49.2 \pm 12.2$ years [not significant]) and male (M:F ratio 40:60 among non-responders and
35:65 among responders [not significant]).

We faced a serious problem of suspicion and mistrust amongst householders, which had two main origins: (a) general mistrust of unknown persons asking to open doors, which derived from the relatively high level of crime; and (b) specific mistrust towards an offer of interest in headache, which was not considered a "serious" medical problem. In order to obtain the confidence of people who were doubtful, surveyors asked for help from neighbours who had been spontaneously willing to participate. This strategy helped significantly to increase the response rate.

The overall number of interviewed subjects was 156 , with the following family structure: 34 (22\%) husbands, $45(29 \%)$ wives, $52(33 \%)$ children, $14(9 \%)$ non-related family members (e.g., daughter- or son-in-law) and 11 (7\%) other relatives.

Of the 104 adults, 47 (45\%) reported headache not related to flu, hangover, cold or head injury in the previous year. Forty-four of these were seen by one of the neurologists, together with 17 of 20 randomly selected subjects who reported no headache (three subjects in each group refused the invitation). Hence the validation of the questionnaire was based on 61 respondents with headache (43 [70\%] females; mean age 42.6 \pm 13.9 years). According to the physician diagnoses, 18 subjects (30\%) had migraine or probable migraine (in the latter case disregarding only the criterion of duration), 23 (38\%) had tension-type headache, $5(8 \%)$ had a combination of migraine and tension-type headache and 15 (24\%) were headachefree. The sensitivity and specificity of the questionnaire for migraine were 0.89 and 0.88 respectively, and for tension-type headache 0.67 and 0.78 . The overall kappa was 0.74. As none of the subjects had any of the trigeminal autonomic cephalalgias, we could not judge the validity of the questionnaire for this diagnostic group.

The analysis of prevalences was based on 93 biologically unrelated subjects (60 [65\%] females, mean age $49.6 \pm 11.5$ years), of whom $45(48 \%)$ had had headache in the previous year (i.e., a current headache disorder). Of this sample, 21 met IHS criteria for migraine or probable migraine (disregarding duration), eight of whom fulfilled all criteria (1-year prevalence: $8.6 \%$ [95\% CI: 2.9-14.3\%]); 19 had tension-type headache (1-year prevalence: $20.4 \%$ [95\% CI: 12.2-28.6\%]); and five had a chronic daily headache syndrome (1-year prevalence: $5.4 \%$ [95\% CI: $1-10.0 \%]$ ), two of whom were overusing acute headache medication. Table 1 shows the demographic and clinical characteristics of patients with migraine, tension-type headache and chronic daily headache. The vast majority of those with headache used simple or combination analgesics for acute treatment of headache. Some used ergots, but none used triptans. None 
Table 1 Demographic and clinical characteristics of patients according to diagnosis

\begin{tabular}{|c|c|c|c|c|c|c|c|c|c|}
\hline $\begin{array}{l}\text { Headache } \\
\text { diagnosis }\end{array}$ & $N$ & Females & $\begin{array}{c}\text { Age } \\
\text { (years) }\end{array}$ & $\begin{array}{c}\text { Time since } \\
\text { onset } \\
\text { (years) }\end{array}$ & $\begin{array}{l}\text { Headache } \\
\text { frequency } \\
(\mathrm{d} / \mathrm{mo})\end{array}$ & $\begin{array}{l}\text { Acute } \\
\text { headache } \\
\text { medication }\end{array}$ & $\begin{array}{l}\text { Overuse of } \\
\text { acute } \\
\text { headache } \\
\text { medication }\end{array}$ & $\begin{array}{l}\text { Preventative } \\
\text { medication }\end{array}$ & $\begin{array}{l}\text { Willingness } \\
\text { to pay }\end{array}$ \\
\hline $\begin{array}{l}\text { Probable or } \\
\text { definite } \\
\text { migraine }\end{array}$ & 21 & $19(91 \%)$ & $46.4 \pm 13.4$ & $13.2 \pm 6.8$ & $5.0 \pm 4.5$ & $\begin{array}{lr}\text { SA: } & 7(33 \%) \\
\text { CA: } & 11(52 \%) \\
\text { E: } \quad 1(5 \%) \\
\text { None: } 2(10 \%)\end{array}$ & $3(14 \%)$ & None & 33 GEL \\
\hline $\begin{array}{l}\text { Definite } \\
\text { migraine }\end{array}$ & 8 & $8(100 \%)$ & $47.6 \pm 9.3$ & $17.4 \pm 6.3$ & $3.5 \pm 2.2$ & $\begin{array}{ll}\text { SA: } & 3(38 \%) \\
\text { CA: } & 4(50 \%) \\
\text { E: } & 1(12 \%)\end{array}$ & $1(12 \%)$ & None & \\
\hline $\begin{array}{l}\text { Tension-type } \\
\text { headache }\end{array}$ & 19 & $13(68 \%)$ & $49.2 \pm 10.8$ & $8.7 \pm 5.7$ & $6.8 \pm 7.0$ & $\begin{array}{l}\text { SA: } 5(26 \%) \\
\text { CA: } 9(47 \%) \\
\text { None: } 5(26 \%)\end{array}$ & $1(45)$ & None & $10 \mathrm{GEL}$ \\
\hline $\begin{array}{l}\text { Chronic daily } \\
\text { headache }\end{array}$ & 5 & $4(80 \%)$ & $52.2 \pm 15.6$ & $7.6 \pm 3.9$ & $21.1 \pm 5.5$ & $\begin{array}{ll}\text { SA: } & 1(20 \%) \\
\text { CA: } & 4(80 \%)\end{array}$ & $2(40 \%)$ & None & 43 GEL \\
\hline
\end{tabular}

$S A$, simple analgesics; $C A$, combination analgesics; $E$, ergot

had preventative medication.

The mean value for WTP was GEL 30.1 18.6 per month (approximately USD 15).

\section{Discussion}

We were able to achieve three main goals of the study: first, to construct a screening questionnaire for migraine and tension-type headache in the Georgian language; second, to use it to perform a pilot study, with plausible results; and third, to learn how to undertake a full epidemiological study in Georgia.

The methodology of the door-to-door interviews worked well. We achieved a response rate of $70 \%$, which can be considered quite high. We faced three main problems. First, we had to earn people's confidence in a situation of general mistrust because of the high level of crime. We therefore performed the study during the warm months of spring, summer and early autumn. We asked people of the neighbourhood who were more enthusiastic for help to gain the confidence of others, and were finally able to achieve a good response rate. The second challenge was the fact that the general population, and even physicians, did not consider headache to be a medical problem. Hence a huge amount of educational work had to be done. The third problem was related to data collection on expenditure. Respondents either did not remember their spending well or were reluctant to disclose this information. It should be said that this a common problem, observed in many other populations and countries. People are mostly not keen to talk about their finances. Our decision to use expenditure data for classifying household socioeconomic status was based on the understanding that they would speak more easily about their expenditure than their income, but this may not have been the case. This issue needs a more careful approach and future exploration before a large epidemiological study is conducted in the region.

The diagnostic questionnaire also worked fairly well: the overall agreement between questionnaire and physician diagnoses was 0.74 , which should be considered as a strong agreement level [10]. The questionnaire showed good sensitivities and specificities for migraine, but less so for tension-type headache, which has fewer positive features. Validity for trigeminal autonomic cephalalgias could not be assessed, but, considering the experience with the German version, we expect that it could be used for screening only and all suspected cases would require formal confirmation. We examined 17 subjects who were headache-negative according to the questionnaire and found two had episodic tension-type headache. This suggests that we missed approximately $10 \%$ of people with a headache disorder, which may not be surprising considering the neglect of headache as a medical problem.

The validation of the questionnaire is not finished. We understand that the numbers of patients and controls so far studied are not sufficient. Hence the validation will be con- 
tinued during the main study. The preliminary data suggest, however, that our questionnaire is comparable in quality with others reported in the international literature. The sensitivity and specificity values are quite similar to those of the German version [3]. Several other validated headache questionnaires exist, most of them constructed as screening instruments for migraine and consisting of 3-8 items based on the IHS criteria [11-18]. Lipton et al. presented a very short screening questionnaire for migraine with only three items and were able to achieve a sensitivity of 0.81 and specificity of 0.75 , with a kappa of 0.68 [14]. More detailed migraine questionnaires have been presented by Kallela et al. [19] and Hagen et al. [20]. The former, for migraine only, obtained a sensitivity of 0.99 and a specificity of 0.96 , whilst the kappa value for the comparison between telephone interview and clinical examination was 0.85 . Questionnaires seeking more than one diagnosis result in considerably lower agreement levels. The questionnaire of Hagen et al. differentiated between migraine, non-migraine headache and chronic daily headache with kappa values of $0.59,0.43$ and 0.44 respectively [20]. The questionnaire suggested by Rasmussen et al. for migraine, episodic tension-type headache and chronic tension-type headache achieved relatively low kappa values of 0.43 , 0.30 and 0.24 respectively [10].

We found the 1-year prevalence of migraine or probable migraine (disregarding duration) to be $21.5 \%$, of definite migraine $8.6 \%$, of tension-type headache $20.4 \%$ and of chronic daily headache $5.4 \%$. We accept that the small sample, from Tbilisi only, does not allow these findings to be considered representative for the whole general population of Georgia, but it is important that the observed prevalences are comparable to those in the international literature [1]. The methodology therefore provided plausible results, and can be used (with some improvements) for a full country-wide epidemiological study.

An interesting finding was that, on average, people within the sample were willing to pay GEL 30 (USD 15) per month for the effective treatment of their headache. This information is very important in the context of public-health interventions. Considering the local prices for over-the-counter analgesics and for generic beta-blockers and valproic acid, and even the cost of some triptans, it is evident that GEL 30 is enough to deliver acute and preventative medication to international standards. The pharmaceutical industry has ignored the potential for drug marketing in this and nearby countries. Not only has this been to the likely disadvantage of people who might have benefited from newer drugs: it may also have been commercially misguided.

The likely impact of the full study from the publichealth perspective should not be underestimated. First, it will provide evidence of the currently unquantified burden of headache in Georgia. Second, it will serve as an example for other countries of the Caucasus, a region with approximately 15 million inhabitants for whom this knowledge is also lacking. Third, it will draw the attention of the medical community, as well as of the general population, to the issue of headache as a common, important but treatable medical problem. Fourth, it will stimulate the teaching of doctors in the region, for whom headache disorders have very low priority. Fifth, it may awaken the pharmaceutical industry to possibilities that can be developed in their and in patients' interests.

\section{References}

1. Stovner L, Hagen K, Jensen R et al (2007) The global burden of headache: a documentation of headache prevalence and disability world-wide. Cephalalgia 27:193-210

2. Steiner T, World Headache Alliance (2004) Lifting the burden: the global campaign against headache. Lancet Neurol 3:204-205

3. Fritsche G, Hueppe M, Kukava M et al (2007) Validation of a German language questionnaire for screening for migraine, tension-type headache and trigeminal autonomic cephalalgias. Headache (in press)
4. Lipton RB, Stewart WF, Diamond S et al (2001) Prevalence and burden of migraine in the United States: data from the American Migraine Study II. Headache 41:646-657

5. Stewart WF, Lipton RB, Celentano DD, Reed ML (1992) Prevalence of migraine headache in the United States. Relation to age, income, race, and other sociodemographic factors. JAMA 267:64-69

6. Headache Classification Subcommittee of the International Headache Society. (2004) The International Classification of Headache Disorders. 2nd edn. Cephalalgia 24[Suppl 1]:9-160
7. Katsarava Z, Obermann M, Yoon M-S et al (2007) Prevalence of cluster headache in a population based sample in Germany. Cephalalgia (in press)

8. Dong H, Kouyate B, Cairns J, Sauerborn R (2005) Inequality in willingness-to-pay for community-based health insurance. Health Policy 72:149-156

9. Bortz J (2005) Statistik für Humanund Sozialwissenschaftler, 6. Aufl. ed. Springer Verlag, Heidelberg

10. Rasmussen BK, Jensen R, Olesen J (1991) Questionnaire versus clinical interview in the diagnosis of headache. Headache 31:290-295 
11. Cady RK, Borchert LD, Spalding W et al (2004) Simple and efficient recognition of migraine with 3-question headache screen. Headache 44:323-327

12. Gervil M, Ulrich V, Olesen J, Russell MB (1998) Screening for migraine in the general population: validation of a simple questionnaire. Cephalalgia 18:342-348

13. Lainez MJ, Dominguez M, Rejas J et al (2005) Development and validation of the Migraine Screen Questionnaire (MS-Q). Headache 45:1328-1338
14. Lipton RB, Dodick D, Sadovsky R et al (2003) A self-administered screener for migraine in primary care: the ID Migraine validation study. Neurology 61:375-382

15. Maizels M, Burchette R (2003) Rapid and sensitive paradigm for screening patients with headache in primary care settings. Headache 43:441-450

16. Marcus DA, Kapelewski C, Jacob RG et al (2004) Validation of a brief nurse-administered migraine assessment tool. Headache 44:328-332

17. Pryse-Phillips W, Aube M, Gawel M et al (2002) A headache diagnosis project. Headache 42:728-737
18. Sheftell FD, Cady RK, Borchert LD et al (2005) Optimizing the diagnosis and treatment of migraine. J Am Acad Nurse Pract 17:309-317

19. Kallela M, Wessman M, Farkkila M (2001) Validation of a migraine-specific questionnaire for use in family studies. Eur J Neurol 8:61-66

20. Hagen K, Zwart JA, Vatten L et al (2000) Head-HUNT: validity and reliability of a headache questionnaire in a large population-based study in Norway. Cephalalgia 20:244-251 